\title{
Uniqueness and weak stability for multi-dimensional transport equations with one-sided Lipschitz coefficient
}

\author{
François Bouchut, François James and Simona Mancini
}

\begin{abstract}
The Cauchy problem for a multidimensional linear transport equation with discontinuous coefficient is investigated. Provided the coefficient satisfies a one-sided Lipschitz condition, existence, uniqueness and weak stability of solutions are obtained for either the conservative backward problem or the advective forward problem by duality. Specific uniqueness criteria are introduced for the backward conservation equation since weak solutions are not unique. A main point is the introduction of a generalized flow in the sense of partial differential equations, which is proved to have unique Jacobian determinant, even though it is itself nonunique.
\end{abstract}

Mathematics Subject Classification (2000): 35F10 (primary); 34A36, 35D05, 35B35 (secondary).

\section{Introduction}

We consider the transport equation

$$
\partial_{t} u+\mathbf{a} \cdot \nabla u=0 \quad \text { in }(0, T) \times \mathbb{R}^{N},
$$

with initial data

$$
u(0, x)=u^{0}(x),
$$

where $\mathbf{a}=\left(\mathbf{a}_{i}(t, x)\right)_{i=1, \ldots, N} \in L^{\infty}\left((0, T) \times \mathbb{R}^{N}\right)$ can have discontinuities. The transport equation (1.1) naturally arises with discontinuous coefficient $\mathbf{a}$ in several applications, together with the conservation equation

$$
\partial_{t} \mu+\operatorname{div}(\mathbf{a} \mu)=0 \quad \text { in }(0, T) \times \mathbb{R}^{N} .
$$

It is well known that both problems are closely related to the notion of characteristics, or flow. The flow $X(s, t, x), 0 \leq s, t \leq T, x \in \mathbb{R}^{N}$, is classically defined by the ODE

$$
\partial_{s} X=\mathbf{a}(s, X(s, t, x)), \quad X(t, t, x)=x .
$$

Pervenuto alla Redazione il 31 marzo 2004. 
Indeed, when a is smooth enough, the flow is uniquely determined by (1.4), and the solutions $u$ to (1.1) and $\mu$ to (1.3) are given respectively by the classical formulæ $u(t, x)=u^{0}(X(0, t, x)), \mu(t, x)=\operatorname{det}\left(\partial_{x} X(0, t, x)\right) \mu^{0}(X(0, t, x))$. The whole theory of characteristics fails if $\mathbf{a}$ is not smooth: the flow is no longer uniquely defined, and the notion of solution to (1.1) or (1.3) has to be reinvestigated.

In the specific case of two-dimensional Hamiltonian transport equations, (1.1) has been solved with continuous (non differentiable) coefficients by Bouchut and Desvillettes [7], and more recently with $L_{\text {loc }}^{p}$ coefficients by Hauray [18]. The general well-posedness theory and the connection between the ODE (1.4) and the PDE (1.1) was investigated by DiPerna and Lions [13] within the framework of renormalized solutions, under the assumption that $\mathbf{a}(t,$.$) lies in$ $W_{\text {loc }}^{1,1}\left(\mathbb{R}^{N}\right)$ and its distributional divergence belongs to $L_{\mathrm{loc}}^{\infty}$. The renormalized approach was extended by Bouchut [6] to the Vlasov equation with $B V$ coefficients, and very recently, Ambrosio [3] gave the full generalization in the same context, when $\mathbf{a}(t,.) \in B V_{\text {loc }}$ and $\operatorname{div} \mathbf{a}(t,$.$) is merely L_{\text {loc }}^{1}$. However, the condition on a which arises very often in applications, the so-called one-sided Lipschitz condition (OSLC)

$$
\exists \alpha \in L_{+}^{1}(0, T) \text { s.t. }\langle\mathbf{a}(t, y)-\mathbf{a}(t, x), y-x\rangle \leq \alpha(t)|y-x|^{2}
$$

for almost every $(t, x, y) \in(0, T) \times \mathbb{R}^{N} \times \mathbb{R}^{N}$, implies only an upper bound on $\operatorname{div} \mathbf{a}$, thus $\operatorname{div} \mathbf{a}$ is not absolutely continuous with respect to the Lebesgue measure. It turns out that in this situation the behaviour of the discontinuities is completely different, and the renormalized approach is not adapted.

For discontinuous a, Filippov's theory [14] gives a generalized definition of a solution $X$ to the ODE (1.4) for a merely bounded coefficient a. Uniqueness, as well as some stability results, are ensured under the OSLC condition (1.5). Notice that, in contrast with the classical theory of ODEs, uniqueness only holds for the forward problem, i.e. for $s>t$. This theory is used to solve differential equations and inclusions, see [15, 4], and also to define generalized characteristics in the context of nonlinear conservation laws, as initiated by Dafermos [12].

The interest of (1.5) lies in the fact that it allows to prove rigorous results in the situation where div $\mathbf{a}$ is a negative measure. This corresponds intuitively to a compressive situation, which arises in many applications in nonlinear hyperbolic equations. The conservation equation (1.3) appears indeed in the study of several degenerate hyperbolic conservation laws where solutions are measures in space, see e.g. Keyfitz and Kranzer [21], LeFloch [22] or Zheng and Majda [32], and the system of pressureless gases, see [10] and the references quoted therein. It is also naturally involved in the context of linearization of conservation laws, see [9] in the one-dimensional setting, [16, 17] for an application in fluid mechanics, [20] in the context of an inverse problem. Several results are to be quoted here, Poupaud and Rascle [27] use the Filippov flow to study the multidimensional equation, Popov and Petrovna [25, 26] give several examples of flows and existence results, both in the one and multidimensional cases. 
The idea we use here to solve the Cauchy problem (1.1)-(1.2) is based on a previous work of Bouchut and James [8], and makes use of the so-called duality solutions. The main idea consists in solving the dual (or adjoint) equation to (1.1)-(1.2), which turns out to be a conservative backward problem

$$
\partial_{t} \pi+\operatorname{div}(\mathbf{a} \pi)=0 \quad \text { in }(0, T) \times \mathbb{R}^{N},
$$

with final data

$$
\pi(T, x)=\pi^{T}(x) .
$$

Indeed a formal computation leads to

$$
\partial_{t}(u \pi)+\operatorname{div}(\mathbf{a} u \pi)=0,
$$

and hence to

$$
\frac{d}{d t} \int u(t, x) \pi(t, x) d x=0 .
$$

It is quite classical now that existence for (1.6)-(1.7) leads to uniqueness for the corresponding direct problem, and this has been successfully used in the nonlinear context, since Oleinik [24], Conway [11], Hoff [19]. However, the lack of uniqueness, and therefore of stability, for weak solutions to (1.6)-(1.7) usually forbids the use of (1.9) as a convenient definition for solutions to the direct problem.

The corner stone in [8] was the introduction of the reversible solutions to the backward problem (1.6), thus defining a class for which existence, uniqueness and weak stability hold. The theory of duality solutions follows then, leading to existence, uniqueness, weak stability for both the transport equation (1.1) and the conservation one (1.3), for one space dimension.

In this paper we generalize this approach to multidimension. However, since we are only able to establish weakly stable uniqueness criterion for the conservative backward problem (1.6)-(1.7), and not for the nonconservative one, we are therefore only able to deal with the forward advective, or nonconservative transport equation. The weak stability of the conservation equation (1.3) is presently out of reach in multidimension. In particular, generalized flows in the sense of PDE, which we call transport flows, are not unique. However, since their Jacobian determinant is unique, they allow us to define a notion of reversible solutions to (1.6). The various characterizations we had at hand in [8] (positivity, renormalization) do not hold here, except if $\pi$ can be expressed as a linear combination of Jacobians of locally Lipschitz functions. However it turns out that this is enough to define duality solutions to the direct equation (1.1), and obtain convenient stability results.

The paper is organized as follows. In Section 2, we collect and prove several useful results on coefficients satisfying the OSLC condition (1.5). Section 3 is the core of the paper, it contains the definition of transport flows and reversible solutions. Next, duality solutions are defined in Section 4, where existence and uniqueness are proved. Stability results are gathered in Section 5. Section 6 emphasizes the fact that transport flows are not necessarily unique, and we prove several technical results about Jacobian determinants in the Appendix. 


\section{Properties of coefficients satisfying the OSLC condition}

This section is devoted to remarkable properties of coefficients satisfying the OSLC condition that are used in the paper. Sharper properties can be found in [2].

Lemma 2.1. If a sequence $\mathbf{a}_{n}$ is bounded in $L^{\infty}\left((0, T) \times \mathbb{R}^{N}\right)$ and satisfies the OSLC condition with some $\alpha_{n}(t)$ uniformly bounded in $L^{1}(0, T)$, then, up to a subsequence, $\mathbf{a}_{n}$ converges in $L^{\infty}-w *$ to $\mathbf{a} \in L^{\infty}\left((0, T) \times \mathbb{R}^{N}\right)$ satisfying the OSLC condition with some $\alpha(t)$ such that $\int_{0}^{T} \alpha(t) d t \leq \liminf \int_{0}^{T} \alpha_{n}(t) d t$.

Proof. We can pass to the limit in (1.5) in the sense of distributions in $(0, T) \times$ $\mathbb{R}^{N} \times \mathbb{R}^{N}$. Then the right-hand side involves some $\hat{\alpha}(t) \in \mathcal{M}_{+}(0, T)$ such that $\alpha_{n} \rightarrow \hat{\alpha}$. We deduce that the required OSLC inequality holds with $\alpha$ the absolutely continuous part of $\hat{\alpha}$.

The OSLC condition implies that div a is bounded from above,

$$
\operatorname{div} \mathbf{a} \leq N \alpha(t) .
$$

This is actually a consequence of the following stronger result.

Lemma 2.2. (i) The OSLC condition (1.5) is equivalent to

$$
\frac{\nabla \mathbf{a}+\nabla \mathbf{a}^{t}}{2} \leq \alpha(t) \mathrm{Id}
$$

for a.e. $t$, in the sense of matrix distributions in $\mathbb{R}^{N}$, which means that

$$
\forall h \in \mathbb{R}^{N} \quad \nabla \mathbf{a} \cdot h \cdot h \leq \alpha(t)|h|^{2}
$$

in the sense of distributions in $\mathbb{R}^{N}$.

(ii) A coefficient $\mathbf{a} \in L^{\infty}$ satisfying the OSLC condition verifies that for a.e. $t$, $\nabla \mathbf{a}+\nabla \mathbf{a}^{t}$ is locally a matrix valued bounded measure in $x$. Moreover, for any bounded convex open subset $C$ of $\mathbb{R}^{N}$,

$$
\forall h \in \mathbb{R}^{N} \quad \int_{C}|\nabla \mathbf{a} \cdot h \cdot h| \leq 2\left(\alpha(t)|C|+\|\mathbf{a}\|_{\infty} \operatorname{diam}(C)^{N-1}\right)|h|^{2} .
$$

Proof. We can consider a fixed time $t$. For (i), assume first that a is smooth in $x$. Then the OSLC condition (1.5) is equivalent to

$$
\langle\mathbf{a}(x+\varepsilon h)-\mathbf{a}(x), \varepsilon h\rangle \leq \alpha|\varepsilon h|^{2}
$$

for any $x, h \in \mathbb{R}^{N}$ and $\varepsilon>0$. Now, since

$$
\langle\mathbf{a}(x+\varepsilon h)-\mathbf{a}(x), \varepsilon h\rangle=\int_{0}^{1}\langle\nabla \mathbf{a}(x+\theta \varepsilon h) \varepsilon h, \varepsilon h\rangle d \theta,
$$


if $\nabla \mathbf{a}(x) \cdot h \cdot h \leq \alpha|h|^{2}$ for all $x, h$, we get obviously (2.5). Conversely, if (2.5) holds, then

$$
\int_{0}^{1}\langle\nabla \mathbf{a}(x+\theta \varepsilon h) h, h\rangle d \theta \leq \alpha|h|^{2},
$$

and by letting $\varepsilon \rightarrow 0$ we recover $\nabla \mathbf{a}(x) \cdot h \cdot h \leq \alpha|h|^{2}$. For the general case $\mathbf{a} \in L^{\infty}$, consider the convolution of $\mathbf{a}$ by a smoothing sequence $\rho_{\varepsilon}(x)$, $\mathbf{a}_{\varepsilon}=\rho_{\varepsilon} * \mathbf{a}$, so that $\mathbf{a}_{\varepsilon} \rightarrow \mathbf{a}$ in $L_{\mathrm{loc}}^{1}\left(\mathbb{R}^{N}\right)$. If a satisfies (1.5), then since

$$
\left\langle\mathbf{a}_{\varepsilon}(y)-\mathbf{a}_{\varepsilon}(x), y-x\right\rangle=\int\langle\mathbf{a}(y-z)-\mathbf{a}(x-z), y-z-(x-z)\rangle \rho_{\varepsilon}(z) d z,
$$

$\mathbf{a}_{\varepsilon}$ also satisfies (1.5) for all $\varepsilon>0$, with the same $\alpha$. Thus by the above proof, $\mathbf{a}_{\varepsilon}$ satisfies $\nabla \mathbf{a}_{\varepsilon} \cdot h \cdot h \leq \alpha|h|^{2}$, and by letting $\varepsilon \rightarrow 0$, this gives (2.3). Conversely, if (2.3) holds, then $\nabla \mathbf{a}_{\varepsilon} \cdot h \cdot h=\rho_{\varepsilon} *(\nabla \mathbf{a} \cdot h \cdot h) \leq \alpha|h|^{2}$ thus by the proof above, $\mathbf{a}_{\varepsilon}$ satisfies (1.5). By letting $\varepsilon \rightarrow 0$, we finally get that a itself satisfies (1.5), and this concludes the proof of (i).

For (ii), we have by (2.3) that $\alpha|h|^{2}-\nabla \mathbf{a} \cdot h \cdot h \geq 0$, thus it is locally a measure. We conclude that $\nabla \mathbf{a} \cdot h \cdot h$ is locally a measure for any $h$, and by finite linear combinations that $\nabla \mathbf{a}+\nabla \mathbf{a}^{t}$ is locally a measure. It remains to prove (2.4). Writing $\nabla \mathbf{a} \cdot h \cdot h=\alpha|h|^{2}-\left(\alpha|h|^{2}-\nabla \mathbf{a} \cdot h \cdot h\right)$, we get

$$
|\nabla \mathbf{a} \cdot h \cdot h| \leq \alpha|h|^{2}+\left(\alpha|h|^{2}-\nabla \mathbf{a} \cdot h \cdot h\right) .
$$

The indicator function of $C$ can be approximated by a function $\varphi(x), \varphi \in$ $C_{c}^{\infty}(C), 0 \leq \varphi \leq 1$. Then

$$
\begin{aligned}
\int\left(\alpha|h|^{2}-\nabla \mathbf{a} \cdot h \cdot h\right) \varphi & =\alpha|h|^{2} \int \varphi+\int \mathbf{a}(x) \cdot h \nabla \varphi(x) \cdot h \\
& \leq \alpha|h|^{2}|C|+\|\mathbf{a}\|_{\infty}|h| \int|\nabla \varphi(x) \cdot h|
\end{aligned}
$$

But the convexity of $C$ enables to find $\varphi$ such that for any direction $h$,

$$
\int|\nabla \varphi \cdot h| \leq 2|h| \operatorname{diam}(C)^{N-1},
$$

and this concludes (ii).

Remark 2.3. By Lemma 3.4 in [6], (2.4) is equivalent to the fact that for any $\omega \subset \subset C$ and any $h \in \mathbb{R}^{N}$ such that $\bar{\omega}+\bar{B}(0,|h|) \subset C$,

$$
\begin{aligned}
& \int_{\omega}|\langle\mathbf{a}(t, x+h)-\mathbf{a}(t, x), h\rangle| d x \\
& \leq 2\left(\alpha(t)|C|+\|\mathbf{a}\|_{\infty} \operatorname{diam}(C)^{N-1}\right)|h|^{2} .
\end{aligned}
$$


Remark 2.4. A coefficient $\mathbf{a} \in L^{\infty}$ satisfying the OSLC condition verifies the local inequalities

$$
\begin{aligned}
& 0 \leq \alpha(t)-\partial_{i} \mathbf{a}_{i} \leq N \alpha(t)-\operatorname{div} \mathbf{a}, \quad i=1, \ldots, N, \\
& \left|\frac{\partial_{j} \mathbf{a}_{i}+\partial_{i} \mathbf{a}_{j}}{2}\right| \leq N \alpha(t)-\operatorname{div} \mathbf{a}, \quad i, j=1, \ldots, N, \quad i \neq j,
\end{aligned}
$$

in the sense of measures. This is because by (2.2), the symmetric matrix valued measure $B=\alpha(t) \mathrm{Id}-\left(\nabla \mathbf{a}+\nabla \mathbf{a}^{t}\right) / 2$ is nonnegative, and thus satisfies $0 \leq B_{i i} \leq \operatorname{tr} B,\left|B_{i j}\right| \leq \operatorname{tr} B$ (for the latter inequality, apply the Cauchy-Schwarz inequality to a regularization $B^{\varepsilon}$ of $\left.B,\left|B_{i j}^{\varepsilon}\right| \leq \sqrt{B_{i i}^{\varepsilon} B_{j j}^{\varepsilon}}\right)$. Another useful inequality is obtained by writing that $B \leq(\operatorname{tr} B)$ Id. Replacing $B$ by its value gives $\alpha \mathrm{Id}-\left(\nabla \mathbf{a}+\nabla \mathbf{a}^{t}\right) / 2 \leq(N \alpha-\operatorname{div} \mathbf{a}) \mathrm{Id}$, or

$$
(\operatorname{div} \mathbf{a}) \operatorname{Id}-\frac{\nabla \mathbf{a}+\nabla \mathbf{a}^{t}}{2} \leq(N-1) \alpha(t) \mathrm{Id} .
$$

Notice in particular that the inequalities (2.12) imply that

$$
\begin{gathered}
\operatorname{div} \mathbf{a} \in L^{1}\left((0, T), L_{\mathrm{loc}}^{p}\left(\mathbb{R}^{N}\right)\right) \quad \text { for some } 1 \leq p \leq \infty \\
\Longrightarrow \quad \nabla \mathbf{a}+\nabla \mathbf{a}^{t} \in L^{1}\left((0, T), L_{\mathrm{loc}}^{p}\left(\mathbb{R}^{N}\right)\right)
\end{gathered}
$$

In the case $1<p<\infty$, we have even $\nabla \mathbf{a} \in L^{1}\left((0, T), L_{\text {loc }}^{p}\left(\mathbb{R}^{N}\right)\right)$, by elliptic regularity since $\Delta \mathbf{a}_{i}=\sum_{j} \partial_{j}\left(\partial_{j} \mathbf{a}_{i}+\partial_{i} \mathbf{a}_{j}\right)-\partial_{i} \operatorname{div} \mathbf{a}$.

We end up this section by pointing out in a simplified context the fact that the OSLC condition (1.5) is in some sense orthogonal to the condition of absolute continuity of the divergence of a that is involved in the renormalized theory, and in particular in the work of Ambrosio [3].

Consider a coefficient $\mathbf{a} \in L^{\infty}$ such that for almost every $t>0, \mathbf{a}(t,.) \in$ $B V_{\text {loc }}\left(\mathbb{R}^{N}\right)$, and the total variation is integrable with respect to time (indeed according to [2], the OSLC condition implies this regularity). Then, fixing the time, the matrix-valued measure $\nabla \mathbf{a}$ can be decomposed as $\nabla \mathbf{a}=\nabla^{a} \mathbf{a}+\nabla^{s} \mathbf{a}$, where $\nabla^{a} \mathbf{a}$ is absolutely continuous and $\nabla^{s} \mathbf{a}$ is singular, with respect to the Lebesgue measure. Denoting by $|\lambda|$ the total variation of a (matrix-valued) measure $\lambda$, one has the polar decomposition $\lambda=M|\lambda|$, with $|M(x)|=1$ for $|\lambda|$-a.e. $x \in \mathbb{R}^{N}$. We apply this to the derivative $\nabla \mathbf{a}$ of $\mathbf{a}$, and make use of Alberti's rank one theorem [1], which asserts that the corresponding $M$ is rank-one, so that

$$
\nabla \mathbf{a}=\eta(x) \otimes \xi(x)|\nabla \mathbf{a}|,
$$

where $\xi$ and $\eta$ are unit vectors in $\mathbb{R}^{N}$. The distributional divergence of $\mathbf{a}$ is therefore given by $\operatorname{div} \mathbf{a}=\langle\xi, \eta\rangle|\nabla \mathbf{a}|$, so that $\operatorname{div} \mathbf{a}$ is absolutely continuous with respect to the Lebesgue measure if and only if $\xi$ and $\eta$ are orthogonal $\left|\nabla^{s} \mathbf{a}\right|$-a.e. As it is recalled by Ambrosio in [3], these results hold true in a very general context, but we wish to consider merely the case where the jump set of 
the function a is (locally) a hypersurface in $\mathbb{R}^{N}$. Then the vector $\xi$ turns out to be the normal to the hypersurface, while $\eta$ is the direction of the jump of a. Thus the usual assumption of absolute continuity of the divergence implies that the jump of $\mathbf{a}$ is tangent to the hypersurface.

If now a satisfies the OSLC condition (instead of having absolutely continuous divergence), then the conclusion is radically different, as the following computation shows. Consider a point $x$ in the hypersurface and a neighborhood $\omega \ni x$. The hypersurface divides $\omega$ in two subsets $\omega_{-}$and $\omega_{+}$, and we take the normal $\xi(x)$ pointing to $\omega_{+}$. We assume that the $\operatorname{limits} \mathbf{a}_{+}(x)=\lim _{y \in \omega_{+}} \mathbf{a}(y)$ and $\mathbf{a}_{-}(x)=\lim _{y \in \omega_{-}} \mathbf{a}(y)$ exist. We rewrite the OSLC condition (1.5) by letting $x$ go to the hypersurface in $\omega_{-}$, and by writing $y=x+\varepsilon h$, for any unit vector $h$ and $\varepsilon>0$. After division by $\varepsilon$, we get

$$
\left\langle\mathbf{a}(x+\varepsilon h)-\mathbf{a}_{-}(x), h\right\rangle \leq \alpha \varepsilon .
$$

Now, if $\langle\xi, h\rangle>0, x+\varepsilon h \in \omega_{+}$for $\varepsilon$ small enough, thus letting $\varepsilon$ go to zero, we obtain

$\left\langle\mathbf{a}_{+}(x)-\mathbf{a}_{-}(x), h\right\rangle \equiv\langle[\mathbf{a}], h\rangle \leq 0$ for any $h$ such that $|h|=1,\langle\xi, h\rangle>0$.

This inequality holds true for any unit vector $h$ such that $\langle\xi, h\rangle=0$ as well, so that [a] has to be colinear with the normal $\xi$. Finally, using (2.17) again we obtain

$$
[\mathbf{a}]=-\lambda \xi \text { for some } \lambda \geq 0 \text {. }
$$

This is radically different from the case above of absolute continuity of the divergence, where $[\mathbf{a}] \cdot \xi=0$.

\section{Backward problem, reversible solutions}

In this section we intend to study weak solutions $\pi \in C\left([0, T], L_{\mathrm{loc}}^{\infty}\left(\mathbb{R}^{N}\right) w *\right)$ to the conservative transport equation (1.6), together with solutions $p \in \operatorname{Lip}_{\text {loc }}$ $\left([0, T] \times \mathbb{R}^{N}\right)$ to the nonconservative transport equation

$$
\partial_{t} p+\mathbf{a} \cdot \nabla p=0 \quad \text { a.e. in }(0, T) \times \mathbb{R}^{N}
$$

We prove very general properties of these solutions, and indeed all that is done in this section is true for a coefficient $\mathbf{a} \in L^{\infty}$ such that there exists a Lipschitz transport flow (see Definition 3.5). Only the existence result Proposition 3.7 makes use of the OSLC condition.

We denote by $\mathcal{W}^{T}$ the vector space of all $\pi \in C\left([0, T], L_{\mathrm{loc}}^{\infty}\left(\mathbb{R}^{N}\right) w *\right)$ solving (1.6) in the weak sense. Recall that there is no uniqueness for the Cauchy problem in $\mathcal{W}^{T}$, so that we introduce various restrictive definitions of convenient solutions. Thus we define several subspaces of $\mathcal{W}^{T}$, and the main goal of this section is to establish inclusions between these subspaces. As we shall see, the definitions do not coincide in the general case. 
The first two notions of solution, and the most natural ones, are defined through sign or cancellation properties. We need to introduce the space $\mathcal{E}^{T}$ of exceptional solutions to the nonconservative equation,

$$
\mathcal{E}^{T}=\left\{p \in \operatorname{Lip}_{\text {loc }}\left([0, T] \times \mathbb{R}^{N}\right) \text { solving (3.1) such that } p(T, .)=0\right\},
$$

and the corresponding open support

$$
V_{e}^{T}=\bigcup_{p_{e} \in \mathcal{E}^{T}}\left\{(t, x) \in(0, T) \times \mathbb{R}^{N} \text { such that } p_{e}(t, x) \neq 0\right\} .
$$

Our first notion of "good" solution to the conservative equation (1.6) is defined via the cancellation in $V_{e}^{T}$,

$$
\mathcal{V}^{T}=\left\{\pi \in \mathcal{W}^{T} \text { such that } \pi=0 \text { a.e. in } V_{e}^{T}\right\} .
$$

Nonnegative solutions will also play a natural part here, thus we define

$$
\mathcal{P}^{T}=\left\{\pi \in \mathcal{W}^{T} ; \pi \geq 0\right\} .
$$

Before giving other definitions, we prove the following preliminary result.

Proposition 3.1. We have $\operatorname{Vect}\left(\mathcal{P}^{T}\right) \subset \mathcal{V}^{T}$, that is any linear combination of nonnegative solutions to (1.6) satisfies the cancellation property (3.4).

The proof relies on the following lemma, which defines some kind of weak product.

Lemma 3.2. Let $\pi \in \mathcal{W}^{T}$ and $p \in \operatorname{Lip}_{\text {loc }}\left([0, T] \times \mathbb{R}^{N}\right)$ solve (3.1). Then $p \pi \in \mathcal{W}^{T}$.

Proof. Let $\pi_{\varepsilon}$ and $(\mathbf{a} \pi)_{\varepsilon}$ denote the approximations of $\pi$ and $\mathbf{a} \pi$ by convolution by a smoothing kernel in $(t, x)$. Since $\partial_{t} \pi_{\varepsilon}+\operatorname{div}(\mathbf{a} \pi)_{\varepsilon}=0$ and using the equation satisfied by $p$ we have

$$
\partial_{t}\left(p \pi_{\varepsilon}\right)+\operatorname{div}\left(p(\mathbf{a} \pi)_{\varepsilon}\right)=\pi_{\varepsilon} \partial_{t} p+(\mathbf{a} \pi)_{\varepsilon} \cdot \nabla p=\left((\mathbf{a} \pi)_{\varepsilon}-\mathbf{a} \pi_{\varepsilon}\right) \cdot \nabla p,
$$

thus letting $\varepsilon \rightarrow 0$ we get

$$
\partial_{t}(p \pi)+\operatorname{div}(\mathbf{a} p \pi)=0 \quad \text { in }(0, T) \times \mathbb{R}^{N} .
$$

Since $p \pi \in C\left([0, T], L_{\mathrm{loc}}^{\infty}\left(\mathbb{R}^{N}\right) w *\right)$, we have the result.

Proof of Proposition 3.1. Let $\pi \in \mathcal{W}^{T}$ such that $\pi \geq 0$. We actually prove that for any any $p_{e} \in \mathcal{E}^{T}$, we have $p_{e} \pi=0$ almost everywhere in $(0, T) \times$ $\mathbb{R}^{N}$. This obviously implies the result. Since $\left|p_{e}\right|$ also belongs to $\mathcal{E}^{T}$ when $p_{e} \in \mathcal{E}^{T}$, we can assume without loss of generality that $p_{e} \geq 0$. Since $p_{e} \pi \in C\left([0, T], L_{\mathrm{loc}}^{\infty}\left(\mathbb{R}^{N}\right) w *\right), p_{e} \pi \geq 0$, and $\left(p_{e} \pi\right)(T,)=$.0 , we conclude with Lemma 3.2 and by integration on cones that $p_{e} \pi \equiv 0$. The conclusion that $\pi \in \mathcal{V}^{T}$ follows obviously by the definition of $\mathcal{V}^{T}$, since each set in the right-hand side of (3.3) is open.

We give now two new definitions of weak solutions to (1.6). The first one takes advantage of an additional conservation law satisfied by the Jacobian matrix of a set of $N$ solutions to the transport problem (3.1). Actually such a property is quite classical in the context of elastodynamics: the Jacobian and cofactor matrices of a velocity field satisfy conservation equations, see for instance Quin [28], or Wagner [31] for a survey in the context of conservation laws. 
Theorem and Definition 3.3. (Jacobian solutions.) Let $p_{i} \in \operatorname{Lip}_{\text {loc }}\left([0, T] \times \mathbb{R}^{N}\right)$, $1 \leq i \leq N$, solve

$$
\partial_{t} p_{i}+\mathbf{a} \cdot \nabla p_{i}=0 \quad \text { a.e. in }(0, T) \times \mathbb{R}^{N} .
$$

Then the Jacobian determinant

$$
\pi=J\left(p_{1}, \ldots, p_{N}\right) \equiv \operatorname{det}\left(\nabla p_{1}, \ldots, \nabla p_{N}\right) \in C\left([0, T], L_{\mathrm{loc}}^{\infty}\left(\mathbb{R}^{N}\right) w *\right)
$$

is a weak solution to (1.6). We shall call such a solution $\pi$ to (1.6) a Jacobian solution, and denote by $\mathcal{J}^{T} \subset \mathcal{W}^{T}$ the set of these solutions.

Proof. Let us apply Lemma A.3 in the Appendix, in the variables $(t, x) \in$ $(0, T) \times \mathbb{R}^{N} \subset \mathbb{R}^{N+1}$, to the Hamiltonians $H_{i}=p_{i}, i=1, \ldots, N$. We get that $\operatorname{div}_{t, x} V=0$, where $V=\left(V_{0}, V_{1}, \ldots, V_{N}\right)$ has to be computed by (A.4). We have $V_{0}=\operatorname{det}\left(\partial p_{i} / \partial x_{j}\right), 1 \leq i, j \leq N$, i.e. $V_{0}=\pi$. Then, for $1 \leq k \leq N$, denoting $p=\left(p_{1}, \ldots, p_{N}\right)$,

$$
\begin{aligned}
V_{k} & =(-1)^{k} \operatorname{det}\left(\partial_{t} p, \frac{\partial p}{\partial x_{1}}, \ldots, \frac{\widehat{\partial p}}{\partial x_{k}}, \ldots, \frac{\partial p}{\partial x_{N}}\right) \\
& =(-1)^{k} \operatorname{det}\left(-\sum_{j=1}^{N} \mathbf{a}_{j} \frac{\partial p}{\partial x_{j}}, \frac{\partial p}{\partial x_{1}}, \ldots, \frac{\widehat{\partial p}}{\partial x_{k}}, \ldots, \frac{\partial p}{\partial x_{N}}\right) \\
& =-\mathbf{a}_{k}(-1)^{k} \operatorname{det}\left(\frac{\partial p}{\partial x_{k}}, \frac{\partial p}{\partial x_{1}}, \ldots, \frac{\widehat{\partial p}}{\partial x_{k}}, \ldots, \frac{\partial p}{\partial x_{N}}\right) \\
& =\mathbf{a}_{k} \pi,
\end{aligned}
$$

which gives (1.6). The time continuity of $\pi$ comes from the weak continuity of the Jacobian, see Lemma A.4. Indeed, if $t_{n} \rightarrow t$, then $p\left(t_{n},.\right) \rightarrow p(t,$.$) locally$ uniformly with uniform bound in $\operatorname{Lip}_{\text {loc }}$ thus $J\left(p\left(t_{n},.\right)\right) \rightarrow J(p(t,)$.$) .$

We have some kind of uniqueness for Jacobian solutions, in the following sense.

Lemma 3.4. Let $p_{i}, q_{i} \in \operatorname{Lip}_{\mathrm{loc}}\left([0, T] \times \mathbb{R}^{N}\right)$, for $i=1, \ldots, N$, solve (3.8), with $p_{i}(T,)=.q_{i}(T,$.$) . Recall that this implies by Theorem 3.3$ that $J(p), J(q) \in \mathcal{W}^{T}$. Assume moreover that $J(p), J(q) \in \mathcal{V}^{T}$ (by Proposition 3.1 this is the case in particular if $J(p) \geq 0$ and $J(q) \geq 0)$. Then $J(p)=J(q)$ almost everywhere.

Proof. On the one hand, $u_{i} \equiv p_{i}-q_{i}$ is a locally Lipschitz solution to the transport equation $(3.1)$, and $u_{i}(T,)=$.0 , i.e. $u_{i} \in \mathcal{E}^{T}$. On the other hand, if we define $v=J(p)$ or $v=J(q)$, we have $v \in \mathcal{V}^{T}$, and by definition, we get $v=0$ a.e. in $\left\{u_{i} \neq 0\right\}$. Now, on the set where $p \neq q$, this gives $v=0$ a.e., thus $J(p)=J(q)=0$. On the set where $p=q$, we have $\nabla p=\nabla q$ a.e. (see for example [6], Theorem 2.1), so that $J(p)=J(q)$ as well. 
The second and last notion of solution we give is the most convenient one from the viewpoint of uniqueness and stability results. However, it does not enjoy really handable characterizations. It is defined through a specific notion of flow, which we introduce now.

Definition 3.5. (Transport flow.) We say that a vector $X^{T} \in \operatorname{Lip}\left([0, T] \times \mathbb{R}^{N}\right)$ is a transport flow if

$$
\partial_{t} X^{T}+\mathbf{a} \cdot \nabla X^{T}=0 \quad \text { in }(0, T) \times \mathbb{R}^{N}, \quad X^{T}(T, x)=x,
$$

and $J\left(X^{T}\right) \geq 0$.

Remark 3.6. The definition requires that $X^{T}$ is globally Lipschitz continuous. This condition ensures that $\left|X^{T}(t, x)-x\right| \leq\left\|\partial_{t} X^{T}\right\|_{L^{\infty}}(T-t)$, and therefore that $X^{T}(t,$.$) tends to infinity at infinity. This estimate can be interpreted as a$ kind of finite speed of propagation property.

We first provide a sufficient condition for the existence of a transport flow.

Proposition 3.7. For any coefficient $\mathbf{a} \in L^{\infty}\left((0, T) \times \mathbb{R}^{N}\right)$ satisfying the OSLC condition, there exists a transport flow $X^{T}$.

Proof. Consider a sequence of coefficients $\mathbf{a}_{n} \in C^{1}, \mathbf{a}_{n}$ bounded in $L^{\infty}$, satisfying the OSLC condition for some $\alpha_{n}$ bounded in $L^{1}$, such that $\mathbf{a}_{n} \rightarrow \mathbf{a}$ in $L_{\text {loc }}^{1}$. Then we have a transport flow associated to $\mathbf{a}_{n}, X_{n}^{T}(t, x)=X_{n}(T, t, x)$ where $X_{n}$ is the classical flow,

$$
\partial_{s} X_{n}(s, t, x)=\mathbf{a}_{n}\left(s, X_{n}(s, t, x)\right), \quad X_{n}(t, t, x)=x .
$$

According to the OSLC condition, we have

$$
\begin{aligned}
& \partial_{s}\left|X_{n}(s, t, y)-X_{n}(s, t, x)\right|^{2} / 2 \\
& =\left\langle\mathbf{a}_{n}\left(s, X_{n}(s, t, y)\right)-\mathbf{a}_{n}\left(s, X_{n}(s, t, x)\right), X_{n}(s, t, y)-X_{n}(s, t, x)\right\rangle \\
& \leq \alpha_{n}(s)\left|X_{n}(s, t, y)-X_{n}(s, t, x)\right|^{2},
\end{aligned}
$$

thus for $0 \leq t \leq s \leq T$,

$$
\left|X_{n}(s, t, y)-X_{n}(s, t, x)\right| \leq|y-x| e^{\int_{t}^{s} \alpha_{n}(\tau) d \tau},
$$

which yields a uniform Lipschitz estimate in $x$ for $X_{n}$,

$$
\operatorname{Lip}\left(X_{n}(s, t, .)\right) \leq e^{\int_{t}^{s} \alpha_{n}(\tau) d \tau} .
$$

But 3.12 yields a Lipschitz estimate in $s$, while the transport equation $\partial_{t} X_{n}+$ $\mathbf{a}_{n} \cdot \nabla X_{n}=0$ yields a Lipschitz estimate in $t$. Therefore, up to a subsequence, $X_{n} \rightarrow X$ locally uniformly. We have that $X(s, t, x)$ is Lipschitz continuous in the domain $0 \leq t \leq s \leq T, x \in \mathbb{R}^{N}, \partial_{t} X+\mathbf{a} \cdot \nabla X=0$ and $X(t, t, x)=x$. By weak continuity of the Jacobian $J\left(X_{n}\right) \rightarrow J(X)$ in $L^{\infty}-w *$, thus $J(X) \geq 0$. We conclude that $X^{T}(t, x)=X(T, t, x)$ is a transport flow. 
We emphasize that, in general, there is no uniqueness in the notion of transport flow, an explicit counterexample is provided in Section 6 below. However, we have the following uniqueness result for the Jacobian.

Proposition 3.8. All possible transport flows $X^{T}$ have the same Jacobian determinant $J\left(X^{T}\right)$.

Proof. If $X^{T}$ is a transport flow, then $J\left(X^{T}\right) \geq 0$, thus by Theorem 3.3 and Proposition 3.1, $J\left(X^{T}\right) \in \mathcal{V}^{T}$. Therefore Lemma 3.4 ensures that $J\left(X^{T}\right)$ is uniquely determined. More precisely, $X^{T}$ is uniquely defined outside $V_{e}^{T}$, thus $J\left(X^{T}\right)$ also. Now, according to Proposition 3.1, we have $J\left(X^{T}\right)=0$ in $V_{e}^{T}$, so that indeed $J\left(X^{T}\right)$ is uniquely determined everywhere.

We are now in position to define the final notion of "good" solutions to (1.6).

Definition 3.9. (Reversible solutions.) We say that $\pi \in \mathcal{W}^{T}$ is a reversible solution to (1.6), and we shall denote $\pi \in \mathcal{R}^{T}$, if for some transport flow $X^{T}$ one has

$$
\pi(t, x)=\pi\left(T, X^{T}(t, x)\right) J\left(X^{T}\right)(t, x)
$$

for all $0 \leq t \leq T$ and a.e. $x \in \mathbb{R}^{N}$.

We notice that this formula is meaningful. Indeed, according to Lemma A.2, at a fixed time $t$, and for any Borel set $Z \subset \mathbb{R}^{N}$ such that $|Z|=0$, we have that $J\left(X^{T}\right)=0$ a.e. where $X^{T} \in Z$. This proves, together with Remark 3.6, that the right-hand side of (3.16) is well-defined as an element of $L_{\text {loc }}^{\infty}\left(\mathbb{R}^{N}\right)$ for each $t$. Also, according to Proposition 3.8, if (3.16) holds for some transport flow $X^{T}$, then it holds for all transport flows. Therefore, $\mathcal{R}^{T}$ is a vector space.

Theorem 3.10. (Conservative backward Cauchy problem.) For any $\pi^{T} \in L_{\mathrm{loc}}^{\infty}\left(\mathbb{R}^{N}\right)$, there exists a unique reversible solution $\pi \in \mathcal{R}^{T}$ to (1.6) such that $\pi(T,)=.\pi^{T}$.

Proof. Uniqueness is obvious from the definition. We claim that a reversible solution with final data $\pi^{T} \in L_{\mathrm{loc}}^{\infty}\left(\mathbb{R}^{N}\right)$ can be obtained by the formula

$$
\pi=\pi^{T}\left(X^{T}\right) J\left(X^{T}\right),
$$

where $X^{T}$ is any transport flow. Indeed, for the same reason as above, $\pi(t,$. is well-defined as an element of $L_{\mathrm{loc}}^{\infty}\left(\mathbb{R}^{N}\right)$ for each $t$. This definition does not depend on the choice of $X^{T}$, and obviously $\pi(T,)=.\pi^{T}$. It only remains to prove that $\pi$ is time continuous and satisfies (1.6) in the weak sense.

Assume first that $\pi^{T}$ is locally Lipschitz continuous. Then $p \equiv \pi^{T}\left(X^{T}\right) \in$ $\operatorname{Lip}_{\text {loc }}\left([0, T] \times \mathbb{R}^{N}\right)$ solves $\partial_{t} p+\mathbf{a} \cdot \nabla p=0$, and by Lemma 3.2 we get that $\pi=p J\left(X^{T}\right) \in \mathcal{W}^{T}$.

In the general case $\pi^{T} \in L_{\text {loc }}^{\infty}$, we can approximate $\pi^{T}$ by $\pi_{n}^{T} \in C^{1}$ such that $\pi_{n}^{T}$ is bounded in $L_{\mathrm{loc}}^{\infty}$ and $\pi_{n}^{T} \rightarrow \pi^{T}$ a.e. Let $Z \subset \mathbb{R}^{N}$ be the set where $\pi_{n}^{T}(x)$ does not converge to $\pi^{T}(x)$, with $|Z|=0$. Then for a fixed $t, \pi_{n}(t, x) \equiv \pi_{n}^{T}\left(X^{T}(t, x)\right) J\left(X^{T}\right)(t, x) \rightarrow \pi^{T}\left(X^{T}(t, x)\right) J\left(X^{T}\right)(t, x)=$ $\pi(t, x)$ a.e., because $J\left(X^{T}\right)(t, x)=0$ a.e. where $X^{T}(t, x) \in Z$. Then, the equation $\partial_{t} \pi_{n}+\operatorname{div}\left(\mathbf{a} \pi_{n}\right)=0$ ensures uniform time continuity, thus $\pi_{n} \rightarrow \pi$ in $C\left([0, T], L_{\mathrm{loc}}^{\infty}\left(\mathbb{R}^{N}\right) w *\right)$ and $\pi$ is a weak solution to (1.6). 
We look now for intrinsic conditions characterizing the reversible solutions, that do not involve any transport flow. It follows from formula (3.17) and from Proposition 3.1 that

$$
\mathcal{R}^{T} \subset \operatorname{Vect}\left(\mathcal{P}^{T}\right) \subset \mathcal{V}^{T} .
$$

In the one space dimension case, these inclusions are equalities, see [8]. Reversibility can also be characterized in terms of renormalization, namely $\pi$ is reversible if and only if $|\pi|$ is a weak solution, and total variation properties. In the present multidimensional context, we are not able to prove the same results, except for the subclass of linear combinations of Jacobian solutions. This is the aim of the following three propositions. Note that this is a difficult problem to decompose a bounded function as a linear combination of Jacobian determinants of Lipschitz functions, see [23].

Proposition 3.11. Let $\pi \in \mathcal{W}^{T}$, such that $\pi(T,$.$) can be written as a linear$ combination of Jacobians of locally Lipschitz functions. Then $\pi$ is reversible if and only if

$$
\pi \in \operatorname{Vect}\left(\mathcal{J}^{T}\right) \cap \mathcal{V}^{T} .
$$

Proof. By assumption, we can write $\pi(T,)=.\sum_{k=1}^{K} \lambda_{k} J\left(p_{k}^{T}\right)$, where $p_{k}^{T} \in$ $\left(\operatorname{Lip}_{\text {loc }}\left(\mathbb{R}^{N}\right)\right)^{N}$. First, if $\pi$ is reversible, then (3.18) gives $\pi \in \mathcal{V}^{T}$, and denoting by $X^{T}$ a transport flow,

$$
\begin{aligned}
\pi(t, .) & =\pi\left(T, X^{T}(t, .)\right) J\left(X^{T}(t, .)\right) \\
& =\sum_{k=1}^{K} \lambda_{k} J\left(p_{k}^{T}\right)\left(X^{T}(t, .)\right) J\left(X^{T}(t, .)\right) \\
& =\sum_{k=1}^{K} \lambda_{k} J\left(p_{k}^{T}\left(X^{T}(t, .)\right)\right) .
\end{aligned}
$$

Since $p_{k} \equiv p_{k}^{T}\left(X^{T}\right)$ is a locally Lipschitz solution to the nonconservative equation, we deduce that $\pi \in \operatorname{Vect}\left(\mathcal{J}^{T}\right) \cap \mathcal{V}^{T}$.

Conversely, let us assume that $\pi \in \operatorname{Vect}\left(\mathcal{J}^{T}\right) \cap \mathcal{V}^{T}$. We have

$$
\pi=\sum_{k=1}^{K} \lambda_{k} J\left(p_{k}\right),
$$

where $p_{k} \in\left(\operatorname{Lip}_{\text {loc }}\left([0, T] \times \mathbb{R}^{N}\right)\right)^{N}$ solves $\partial_{t} p_{k}+\mathbf{a} \cdot \nabla p_{k}=0$. Consider the unique reversible solution $\pi_{k}^{\text {rev }}$ to $(1.6)$ with final data $J\left(p_{k}(T,).\right)$. In other words, $\pi_{k}^{r e v}=J\left(p_{k}(T,).\right)\left(X^{T}\right) J\left(X^{T}\right)=J\left(q_{k}\right)$, with $q_{k}=p_{k}\left(T, X^{T}\right)$. Define $\pi^{\text {rev }}=\sum_{k} \lambda_{k} \pi_{k}^{\text {rev }}$. Then, since $p_{k}-q_{k} \in \mathcal{E}^{T}$, for $(t, x) \notin V_{e}^{T}$ we have $p_{k}(t, x)=q_{k}(t, x)$ thus $\nabla p_{k}=\nabla q_{k}$ a.e. in $\left(V_{e}^{T}\right)^{c}$ and $J\left(p_{k}\right)=J\left(q_{k}\right)$ a.e. in $\left(V_{e}^{T}\right)^{c}$ for all $k$. We conclude that $\pi(t, x)=\pi^{\text {rev }}(t, x)$ a.e. in $\left(V_{e}^{T}\right)^{c}$. But since $\pi, \pi^{r e v} \in \mathcal{V}^{T}, \pi=0=\pi^{r e v}$ a.e. in $V_{e}^{T}$, thus finally $\pi=\pi^{r e v}$ a.e. in $(0, T) \times \mathbb{R}^{N}$. 
Proposition 3.12. Let $\pi \in \operatorname{Vect}\left(\mathcal{J}^{T}\right)$. Then $\pi \in \mathcal{R}^{T}$ if and only if $|\pi| \in \mathcal{W}^{T}$.

Proof. We first notice from (3.17) that if $\pi \in \mathcal{R}^{T}$, then $|\pi| \in \mathcal{R}^{T} \subset \mathcal{W}^{T}$. Conversely, if $|\pi| \in \mathcal{W}^{T}$, then by Proposition 3.1, $|\pi| \in \mathcal{V}^{T}$. By definition of $\mathcal{V}^{T}$, we deduce that $\pi \in \mathcal{V}^{T}$, and by Proposition 3.11, $\pi \in \mathcal{R}^{T}$.

Remark 3.13. Any $\pi \in \mathcal{W}^{T}$ satisfies $\pi \in C\left([0, T], L_{\text {loc }}^{1}\left(\mathbb{R}^{N}\right)\right)$, and in particular $|\pi| \in C\left([0, T], L_{\mathrm{loc}}^{\infty}\left(\mathbb{R}^{N}\right) w *\right)$. This is the consequence of Lemma 2.2 and of Theorem 3.3 in [6].

Proposition 3.14. For any $\pi \in \mathcal{W}^{T}$, the application

$$
t \mapsto\|\pi(t, \cdot)\|_{L^{1}\left(\mathbb{R}^{N}\right)} \in[0, \infty]
$$

is nonincreasing in $[0, T]$. Moreover,

(i) If $\pi$ is reversible then this function is constant.

(ii) If $\pi \in \operatorname{Vect}\left(\mathcal{J}^{T}\right)$ and if the above function is constant and finite, then $\pi$ is reversible.

Proof. According to Lemma 3.2, for any $p \in \operatorname{Lip}_{\text {loc }}\left([0, T] \times \mathbb{R}^{N}\right)$ solving (3.1), we have $p \pi \in \mathcal{W}^{T}$. Take any transport flow $X^{T}$, and choose $p(t, x)=$ $\varphi\left(X^{T}(t, x)\right)$, with $\varphi \in C_{c}^{1}\left(\mathbb{R}^{N}\right)$. Taking into account Remark 3.6 and integrating the equation satisfied by $p \pi$, we get for any $0 \leq t \leq T$

$$
\int_{\mathbb{R}^{N}} \varphi(x) \pi(T, x) d x=\int_{\mathbb{R}^{N}} \varphi\left(X^{T}(t, x)\right) \pi(t, x) d x .
$$

This means that $\pi(T,) d$.$x is the image of \pi(t,) d$.$x by the function x \mapsto$ $X^{T}(t, x)$. In particular, (3.23) is valid also for all $\varphi$ measurable and bounded with compact support. Then, taking $\varphi=\psi \operatorname{sgn}(\pi(T,)$.$) , we get for any \psi$ measurable bounded with compact support and nonnegative

$$
\int_{\mathbb{R}^{N}} \psi(x)|\pi(T, x)| d x \leq \int_{\mathbb{R}^{N}} \psi\left(X^{T}(t, x)\right)|\pi(t, x)| d x .
$$

Letting $\psi \rightarrow 1$ we conclude that

$$
\int_{\mathbb{R}^{N}}|\pi(T, x)| d x \leq \int_{\mathbb{R}^{N}}|\pi(t, x)| d x .
$$

Since the restriction on a subinterval of any weak solution $\pi$ to (1.6) is again a weak solution, we can replace $T$ in (3.25) by any value $s \geq t$, which proves the first assertion.

In order to prove (i), we first recall that if $\pi \in \mathcal{R}^{T}$, then $|\pi| \in \mathcal{R}^{T}$. Thus in the case where $\pi(T,.) \in L_{c}^{\infty}\left(\mathbb{R}^{N}\right)$, we obviously get that $\int|\pi(t, x)| d x$ is constant by integrating the equation satisfied by $|\pi|$. In the general case $\pi(T,.) \in L_{\mathrm{loc}}^{\infty}$, we can define $\pi_{n}^{T}(x)=\pi(T, x) \mathbb{1}_{|x| \leq n}$. Denoting by $\pi_{n}$ the reversible solution with final data $\pi_{n}^{T}$, we have that $\pi_{n}(t,.) \rightarrow \pi(t,$.$) a.e. for$ each $t$, and we conclude by monotone convergence. 
Let us finally prove (ii). Consider the reversible solution $\pi^{r e v}$ with final data $\pi(T,$.$) . Since \pi \in \operatorname{Vect}\left(\mathcal{J}^{T}\right)$, we have by the same argument as in the proof of Proposition 3.11 that $\pi=\pi^{r e v}$ a.e. in $\left(V_{e}^{T}\right)^{c}$. Thus for almost every $t$,

$$
\int_{\mathbb{R}^{N}}|\pi(t, x)| d x=\int_{(t, x) \notin V_{e}^{T}}\left|\pi^{r e v}(t, x)\right| d x+\int_{(t, x) \in V_{e}^{T}}|\pi(t, x)| d x .
$$

But since $\pi^{r e v}=0$ a.e. in $V_{e}^{T}$, and by (i), we have

$$
\int_{(t, x) \notin V_{e}^{T}}\left|\pi^{r e v}(t, x)\right| d x=\int_{\mathbb{R}^{N}}\left|\pi^{r e v}(t, x)\right| d x=\int_{\mathbb{R}^{N}}|\pi(T, x)| d x .
$$

Therefore, writing that $\int|\pi(t, x)| d x$ is constant and finite, we obtain that $\int_{(t, x) \in V_{e}^{T}}|\pi(t, x)| d x=0$ for a.e. $t$, which gives that $\pi=0$ a.e. in $V_{e}^{T}$. We conclude that $\pi=\pi^{r e v}$ a.e. in $(0, T) \times \mathbb{R}^{N}$.

\section{Forward problem, duality solutions}

We consider now the forward nonconservative transport problem (1.1)-(1.2). We can only hope to have solutions of bounded variation in $x$, thus we define the space

$$
\mathcal{S}_{B V}=C\left([0, T], L_{\mathrm{loc}}^{1}\left(\mathbb{R}^{N}\right)\right) \cap \mathcal{B}\left([0, T], B V_{\mathrm{loc}}\left(\mathbb{R}^{N}\right)\right),
$$

where $\mathcal{B}$ stands for the space of bounded functions. This regularity is however not enough to ensure a unique a priori determination of the product $\mathbf{a} \times \nabla u$. We therefore have to define solutions in a weak sense, via the backward conservative problem studied in the previous section. Reversible solutions on a subinterval $[0, \tau]$ are involved, which is meaningful, $T$ has to be replaced by $\tau$ in Definitions 3.9 and 3.5 .

Definition 4.1. (Duality solutions.) We say that $u \in \mathcal{S}_{B V}$ is a duality solution to (1.1) if for any $0<\tau \leq T$ and for any reversible solution $\pi$ to $\partial_{t} \pi+\operatorname{div}(\mathbf{a} \pi)=0$ in $(0, \tau) \times \mathbb{R}^{N}$ with compact support in $x$, one has that

$$
t \longmapsto \int_{\mathbb{R}^{N}} u(t, x) \pi(t, x) d x \text { is constant in }[0, \tau]
$$

This definition is motivated by the following lemma.

Lemma 4.2. Let $p \in \operatorname{Lip}_{\text {loc }}\left([0, T] \times \mathbb{R}^{N}\right)$ solve $\partial_{t} p+\mathbf{a} \cdot \nabla p=0$ a.e. in $(0, T) \times \mathbb{R}^{N}$. Then $p$ is a duality solution.

Proof. By Lemma 3.2, for any reversible solution $\pi$ in $(0, T) \times \mathbb{R}^{N}$, we have $\partial_{t}(p \pi)+\operatorname{div}(\mathbf{a} p \pi)=0$. If $\pi$ has compact support, we deduce by integration in $x$ that $\int p(t, x) \pi(t, x) d x=c s t$ in $[0, T]$. Since $p$ is also a solution on a subinterval $[0, \tau]$, this yields the result. 
We are now in position to prove the main result of this section, namely existence and uniqueness in the duality sense for the transport equation (1.1).

Theorem 4.3. (Nonconservative forward Cauchy problem.) For any $u^{0} \in B V_{\text {loc }}\left(\mathbb{R}^{N}\right)$, there exists a unique duality solution $u \in \mathcal{S}_{B V}$ to $(1.1)$ such that $u(0,)=.u^{0}$.

Proof of uniqueness. Assume that there exists a duality solution $u$ such that $u(0,)=$.0 . Then, by definition, we have for any $0 \leq t \leq \tau$

$$
\int_{\mathbb{R}^{N}} u(t, x) \pi(t, x) d x=\int_{\mathbb{R}^{N}} u(0, x) \pi(0, x) d x=0,
$$

for any $\pi$ reversible solution in $[0, \tau]$ with compact support. Choosing in particular $t=\tau$, and since $\pi(\tau,$.$) is arbitrary in L_{c}^{\infty}$, we obtain that $u(\tau,)=$.0 . This is true for any $0<\tau \leq T$, thus $u \equiv 0$.

The existence proof makes use of the following a priori $B V$ bound.

Lemma 4.4. Assume that $\mathbf{a} \in C^{1}\left([0, T] \times \mathbb{R}^{N}\right)$ and that $u \in C^{2}\left([0, T] \times \mathbb{R}^{N}\right)$ solves (1.1) in the classical sense. Then for any $0 \leq t \leq T$,

$$
\begin{aligned}
& \int_{B\left(x_{0}, R\right)} \sum_{i=1}^{N}\left|\frac{\partial u}{\partial x_{i}}(t, x)\right| d x \\
& \leq \sqrt{N} e^{(N-1) \int_{0}^{t} \alpha(s) d s} \int_{B\left(x_{0}, R+t\|\mathbf{a}\|_{\infty}\right)} \sum_{i=1}^{N}\left|\frac{\partial u}{\partial x_{i}}(0, x)\right| d x .
\end{aligned}
$$

Proof. Differentiating (1.1) with respect to $x_{j}$, we get

$$
\left(\partial_{t}+\mathbf{a} \cdot \nabla\right)\left(\partial_{j} u\right)+\partial_{j} \mathbf{a} \cdot \nabla u=0
$$

Then, defining $\psi=\left(\sum_{j}\left(\partial_{j} u\right)^{2}\right)^{1 / 2}$, we obtain

$$
\psi\left(\partial_{t}+\mathbf{a} \cdot \nabla\right) \psi+\sum_{i, j} \partial_{j} \mathbf{a}_{i} \partial_{i} u \partial_{j} u=0,
$$

or

$$
\psi\left(\partial_{t} \psi+\operatorname{div}(\mathbf{a} \psi)\right)+\nabla u^{t} \nabla \mathbf{a} \nabla u-\psi^{2} \operatorname{div} \mathbf{a}=0 .
$$

Noticing that $\nabla u^{t} \nabla u=\psi^{2}$ and

$$
\psi^{2} \operatorname{div} \mathbf{a}-\nabla u^{t} \nabla \mathbf{a} \nabla u=\nabla u^{t}\left((\operatorname{div} \mathbf{a}) \operatorname{Id}-\frac{\nabla \mathbf{a}+\nabla \mathbf{a}^{t}}{2}\right) \nabla u
$$

the equation (4.7) gives with the inequality (2.13)

$$
\psi\left(\partial_{t} \psi+\operatorname{div}(\mathbf{a} \psi)\right) \leq(N-1) \alpha(t) \psi^{2},
$$


and therefore

$$
\partial_{t} \psi+\operatorname{div}(\mathbf{a} \psi) \leq(N-1) \alpha(t) \psi .
$$

Defining $\phi=\psi e^{-(N-1) \int_{0}^{t} \alpha}$, we deduce that

$$
\partial_{t} \phi+\operatorname{div}(\mathbf{a} \phi) \leq 0
$$

and integrating this over a cone yields

$$
\int_{B\left(x_{0}, R\right)} \phi(t, x) d x \leq \int_{B\left(x_{0}, R+t\|\mathbf{a}\|_{\infty}\right)} \phi(0, x) d x .
$$

Translating this to $\psi$ gives

$$
\int_{B\left(x_{0}, R\right)} \psi(t, x) d x \leq e^{(N-1) \int_{0}^{t} \alpha} \int_{B\left(x_{0}, R+t\|\mathbf{a}\|_{\infty}\right)} \psi(0, x) d x .
$$

But according to the Cauchy-Schwarz inequality $\psi \leq \sum\left|\partial_{j} u\right| \leq \sqrt{N} \psi$, which gives the estimate (4.4).

Remark 4.5. In the case $N=1$, Lemma 4.4 reduces to a TVD property.

Proof of existence in Theorem 4.3. Consider a sequence of coefficients $\mathbf{a}_{n} \in C^{2}$, $\mathbf{a}_{n}$ bounded in $L^{\infty}$, satisfying the OSLC condition for some $\alpha_{n}$ bounded in $L^{1}$, such that $\mathbf{a}_{n} \rightarrow \mathbf{a}$ in $L_{\text {loc }}^{1}$. Then we have a classical flow $X_{n}(s, t, x) \in C^{2}$ associated to $\mathbf{a}_{n}$, satisfying $\partial_{s} X_{n}(s, t, x)=\mathbf{a}_{n}\left(s, X_{n}(s, t, x)\right), X_{n}(t, t, x)=x$. Consider also a sequence of initial data $u_{n}^{0} \in C^{2}$, bounded in $B V_{\text {loc }}$, such that $u_{n}^{0} \rightarrow u^{0}$ in $L_{\text {loc }}^{1}$. Then, let us define $u_{n}(t, x) \in C^{2}$ to be the classical solution to

$$
\partial_{t} u_{n}+\mathbf{a}_{n} \cdot \nabla u_{n}=0 \quad \text { in }(0, T) \times \mathbb{R}^{N}, \quad u_{n}(0, .)=u_{n}^{0} .
$$

According to Lemma $4.4, u_{n}$ is uniformly bounded in $\mathcal{B}\left([0, T], B V_{\mathrm{loc}}\left(\mathbb{R}^{N}\right)\right)$. But since $u_{n}$ is given by $u_{n}(t, x)=u_{n}^{0}\left(X_{n}(0, t, x)\right)$, we have for any $0<\tau \leq T$, $\varphi \in L_{c}^{\infty}\left(\mathbb{R}^{N}\right)$ and $0 \leq t \leq \tau$

$$
\begin{aligned}
& \int_{\mathbb{R}^{N}} u_{n}(t, x) \varphi\left(X_{n}(\tau, t, x)\right) J\left(X_{n}\right)(\tau, t, x) d x \\
& =\int_{\mathbb{R}^{N}} u_{n}\left(t, X_{n}(t, \tau, x)\right) \varphi(x) d x \\
& =\int_{\mathbb{R}^{N}} u_{n}^{0}\left(X_{n}(0, \tau, x)\right) \varphi(x) d x \\
& =\int_{\mathbb{R}^{N}} u_{n}^{0}(x) \varphi\left(X_{n}(\tau, 0, x)\right) J\left(X_{n}\right)(\tau, 0, x) d x .
\end{aligned}
$$


But as in the proof of Proposition 3.7, $X_{n}$ is uniformly Lipschitz continuous in the domain $0 \leq t \leq s \leq T, x \in \mathbb{R}^{N}$, and up to a subsequence it converges to $X$, such that $X^{\tau} \equiv X(\tau, .,$.$) is a transport flow in [0, \tau]$. Taking $t=\tau$ in (4.15), we get that $\left|\int u_{n}(\tau,.) \varphi\right| \leq C\|\varphi\|_{\infty}$, the constant $C$ depending on the support of $\varphi$. Therefore $u_{n}$ is bounded in $C\left([0, T], L_{\text {loc }}^{1}\left(\mathbb{R}^{N}\right)\right)$ also. But by (4.14), $u_{n}$ is equicontinuous in time, thus extracting a subsequence again, $u_{n} \rightarrow u$ in $C\left([0, T], L_{\text {loc }}^{1}\left(\mathbb{R}^{N}\right)\right)$, with $u \in \mathcal{S}_{B V}$ satisfying $u(0,)=.u^{0}$. For any $\varphi \in C_{c}\left(\mathbb{R}^{N}\right)$ we can pass to the limit in (4.15), thus for $0 \leq t \leq \tau$

$$
\begin{aligned}
& \int_{\mathbb{R}^{N}} u(t, x) \varphi(X(\tau, t, x)) J(X)(\tau, t, x) d x \\
& =\int_{\mathbb{R}^{N}} u^{0}(x) \varphi(X(\tau, 0, x)) J(X)(\tau, 0, x) d x .
\end{aligned}
$$

By approximation this is still valid for $\varphi \in L_{c}^{\infty}$. Noticing that $\pi(t, x) \equiv$ $\varphi(X(\tau, t, x)) J(X)(\tau, t, x)$ is the reversible solution in $[0, \tau]$ with final data $\varphi$, we conclude that (4.2) holds, and $u$ is a duality solution.

\section{Weak stability}

To have weak stability results under sharp assumptions is a key point in developing an efficient well-posedness theory. We show here that our notions of reversible and duality solutions are very well adapted to this achievement.

We shall consider in this section a sequence of coefficients $\mathbf{a}_{n}$ such that

$$
\mathbf{a}_{n} \text { is uniformly bounded in } L^{\infty}\left((0, T) \times \mathbb{R}^{N}\right),
$$

and

$\mathbf{a}_{n}$ satisfies an OSLC condition for some $\alpha_{n}$ bounded in $L^{1}(0, T)$.

Then, after extraction of a subsequence,

$$
\mathbf{a}_{n} \rightarrow \mathbf{a} \text { in } L^{\infty} w *
$$

and according to Lemma 2.1, a also satisfies an OSLC condition. The main two results of this section are the following.

Theorem 5.1. (Reversible backward stability.) Assume (5.1)-(5.3), and let $\pi_{n}^{T}$ be a bounded sequence in $L_{\mathrm{loc}}^{\infty}\left(\mathbb{R}^{N}\right)$ such that $\pi_{n}^{T} \rightarrow \pi^{T}$ locally in $L^{\infty} w *$. Then the reversible solution $\pi_{n}$ to

$$
\partial_{t} \pi_{n}+\operatorname{div}\left(\mathbf{a}_{n} \pi_{n}\right)=0 \text { in }(0, T) \times \mathbb{R}^{N}, \quad \pi_{n}(T, .)=\pi_{n}^{T}
$$

converges in $C\left([0, T], L_{\mathrm{loc}}^{\infty}\left(\mathbb{R}^{N}\right) w *\right)$ to the reversible solution $\pi$ to

$$
\partial_{t} \pi+\operatorname{div}(\mathbf{a} \pi)=0 \text { in }(0, T) \times \mathbb{R}^{N}, \quad \pi(T, .)=\pi^{T} .
$$

Moreover, $\mathbf{a}_{n} \pi_{n} \rightarrow \mathbf{a} \pi$. 
Theorem 5.2. (Forward duality stability.) Assume (5.1)-(5.3), and let $u_{n}^{0}$ be a bounded sequence in $B V_{\mathrm{loc}}\left(\mathbb{R}^{N}\right)$ such that $u_{n}^{0} \rightarrow u^{0}$ in $L_{\mathrm{loc}}^{1}$. Then the duality solution $u_{n}$ to

$$
\partial_{t} u_{n}+\mathbf{a}_{n} \cdot \nabla u_{n}=0 \quad \text { in } \quad(0, T) \times \mathbb{R}^{N}, \quad u_{n}(0, .)=u_{n}^{0}
$$

converges in $C\left([0, T], L_{\mathrm{loc}}^{1}\left(\mathbb{R}^{N}\right)\right)$ to the duality solution $u$ to

$$
\partial_{t} u+\mathbf{a} \cdot \nabla u=0 \quad \text { in } \quad(0, T) \times \mathbb{R}^{N}, \quad u(0, .)=u^{0} .
$$

Proof of Theorem 5.1. The sequence $\pi_{n}$ is bounded in $L_{\mathrm{loc}}^{\infty}\left(\mathbb{R}^{N}\right)$, uniformly in $t$ and $n$. Since (5.4) gives compactness in time, we have after extraction of a subsequence that $\pi_{n}$ converges to some $\pi$ in $C\left([0, T], L_{\text {loc }}^{\infty}\left(\mathbb{R}^{N}\right) w *\right)$. We have that

$$
\pi_{n}(t, x)=\pi_{n}^{T}\left(X_{n}^{T}(t, x)\right) J\left(X_{n}^{T}\right)(t, x),
$$

where $X_{n}^{T}$ is a transport flow associated to $\mathbf{a}_{n}$. According to (3.15), we can choose $X_{n}^{T}$ uniformly bounded in $\operatorname{Lip}\left([0, T] \times \mathbb{R}^{N}\right)$, and thus we can extract a subsequence converging locally uniformly to some $X^{T} \in \operatorname{Lip}\left([0, T] \times \mathbb{R}^{N}\right)$. Taking into account Lemma 2.2, we have that $\operatorname{div} \mathbf{a}_{n}$ is bounded in $\mathcal{M}_{\mathrm{loc}}((0, T) \times$ $\mathbb{R}^{N}$ ). Thus for $i=1, \ldots, N$,

$$
\begin{aligned}
& \mathbf{a}_{n} \cdot \nabla\left(X_{n}^{T}\right)_{i}=\operatorname{div}\left(\mathbf{a}_{n}\left(X_{n}^{T}\right)_{i}\right)-\operatorname{div}\left(\mathbf{a}_{n}\right)\left(X_{n}^{T}\right)_{i} \\
& \quad \longrightarrow \operatorname{div}\left(\mathbf{a}\left(X^{T}\right)_{i}\right)-\operatorname{div}(\mathbf{a})\left(X^{T}\right)_{i}=\mathbf{a} \cdot \nabla\left(X^{T}\right)_{i},
\end{aligned}
$$

and we deduce that $X^{T}$ is a transport flow associated to a. According to Remark 3.6 and the uniform bounds, when localizing in $x$ in (5.8), only values of $\pi_{n}^{T}$ on a bounded set are involved. Therefore, we can apply Lemma A4 to pass to the limit in (5.8) at a fixed time $t$, and we conclude that $\pi(t, x)=$ $\pi^{T}\left(X^{T}(t, x)\right) J\left(X^{T}\right)(t, x)$, i.e. that $\pi$ is the reversible solution to (5.5). The uniqueness of the limit ensures that in fact it is not necessary to extract any subsequence.

Let us finally prove the convergence of $\mathbf{a}_{n} \pi_{n}$. According to (5.8) and to (3.10), we have

$$
\left(\mathbf{a}_{n}\right)_{k} \pi_{n}=\pi_{n}^{T}\left(X_{n}^{T}\right)(-1)^{k} \operatorname{det}\left(\partial_{t} X_{n}^{T}, \frac{\partial X_{n}^{T}}{\partial x_{1}}, \ldots, \frac{\widehat{\partial X_{n}^{T}}}{\partial x_{k}}, \ldots, \frac{\partial X_{n}^{T}}{\partial x_{N}}\right) .
$$

Applying Lemma A.4 in the variables $(t, x)$, we conclude that we can pass to the limit weakly in the right-hand side, which gives that $\mathbf{a}_{n} \pi_{n} \rightarrow \mathbf{a} \pi$.

Remark 5.3. Another way of proving the convergence of $\mathbf{a}_{n} \pi_{n}$ is by using Lemma 2.2. From the identity $\Delta \mathbf{a}_{i}=\sum_{j} \partial_{j}\left(\partial_{j} \mathbf{a}_{i}+\partial_{i} \mathbf{a}_{j}\right)-\partial_{i}$ div $\mathbf{a}$ and the fact that $\nabla \mathbf{a}_{n}+\nabla \mathbf{a}_{n}^{t}$ is bounded in $\mathcal{M}_{\text {loc }}\left((0, T) \times \mathbb{R}^{N}\right)$, we deduce that $\mathbf{a}_{n}$ is compact in $x$, i.e. that $\left\|\mathbf{a}_{n}(t, x+h)-\mathbf{a}_{n}(t, x)\right\|_{L^{1}\left((0, T) \times B_{R}\right)} \rightarrow 0$ as $h$ tends to 0 , uniformly in $n$. Since $\pi_{n} \rightarrow \pi$ in $C\left([0, T], L_{\text {loc }}^{\infty}\left(\mathbb{R}^{N}\right) w *\right)$, this is enough to conclude that $\mathbf{a}_{n} \pi_{n} \rightarrow \mathbf{a} \pi$. 
Remark 5.4. According to Lemma 2.2 and to Theorem 3.3 in [6], $\pi_{n}$ and $\pi$ lie in $C\left([0, T], L_{\text {loc }}^{1}\left(\mathbb{R}^{N}\right)\right)$. It is an open problem to prove that if $\pi_{n}^{T} \rightarrow \pi^{T}$ in $L_{\mathrm{loc}}^{1}$, then $\pi_{n} \rightarrow \pi$ in $C\left([0, T], L_{\mathrm{loc}}^{1}\left(\mathbb{R}^{N}\right)\right)$.

Proof of Theorem 5.2. The sequence $u_{n}$ is bounded in $\mathcal{S}_{B V}$, and equicontinuous in time (even if (5.6) does not hold in the classical sense, the a priori estimate on $\partial_{t} u_{n}$ in $L_{t}^{\infty}\left(\mathcal{M}_{\mathrm{loc}}\left(\mathbb{R}^{N}\right)\right)$ is valid). Therefore, after extraction of a subsequence, $u_{n}$ converges in $C\left([0, T], L_{\mathrm{loc}}^{1}\left(\mathbb{R}^{N}\right)\right)$ to some $u \in \mathcal{S}_{B V}$ satisfying $u(0,)=.u^{0}$. Now, for any $0<\tau \leq T$ and any $\pi^{\tau} \in L_{c}^{\infty}\left(\mathbb{R}^{N}\right)$, we have that

$$
t \longmapsto \int_{\mathbb{R}^{N}} u_{n}(t, x) \pi_{n}(t, x) d x \text { is constant in }[0, \tau],
$$

where $\pi_{n}$ is the reversible solution to $\partial_{t} \pi_{n}+\operatorname{div}\left(\mathbf{a}_{n} \pi_{n}\right)=0$ in $(0, \tau) \times \mathbb{R}^{N}$, $\pi_{n}(\tau,)=.\pi^{\tau}$. Applying Theorem 5.1 on $(0, \tau)$, we deduce that $\pi_{n} \rightarrow \pi$ in $C\left([0, \tau], L_{\mathrm{loc}}^{\infty}\left(\mathbb{R}^{N}\right) w *\right)$ where $\pi$ is the reversible solution to $\partial_{t} \pi+\operatorname{div}(\mathbf{a} \pi)=0$ in $(0, \tau) \times \mathbb{R}^{N}, \pi(\tau,)=.\pi^{\tau}$. Passing to the limit in (5.11), we get that

$$
t \longmapsto \int_{\mathbb{R}^{N}} u(t, x) \pi(t, x) d x \text { is constant in }[0, \tau],
$$

which means that $u$ is the duality solution to (5.7). By uniqueness, we conclude that it is indeed not necessary to extract any subsequence.

\section{Nonuniqueness of transport flows}

The aim of this section is to show that the uniqueness results established in Section 3 are optimal, in the sense that first the transport flow is not unique, and second its Jacobian is uniquely determined.

Our example is in two space dimensions, and is really the simplest nontrivial coefficient we can think of,

$$
\mathbf{a}\left(t, x_{1}, x_{2}\right)=\left(-\operatorname{sgn} x_{1}, 0\right) .
$$

It is bounded, and obviously satisfies the OSLC condition with $\alpha \equiv 0$. Since the second component of a vanishes identically, the second variable $x_{2}$ only stands as a parameter in (1.6) and (3.1). We deduce the general solution $p \in \operatorname{Lip}_{\text {loc }}\left([0, T] \times \mathbb{R}^{N}\right)$ to (3.1) by just adding a parameter $x_{2}$ to the general solution of the one-dimensional problem obtained in [8],

$$
p\left(t, x_{1}, x_{2}\right)=\varphi\left(\left(\left|x_{1}\right|-(T-t)\right)_{+} \operatorname{sgn} x_{1}, x_{2}\right)+h\left(\left(T-t-\left|x_{1}\right|\right)_{+}, x_{2}\right),
$$

with

$$
\varphi \in \operatorname{Lip}_{\text {loc }}(\mathbb{R} \times \mathbb{R}), \quad h \in \operatorname{Lip}_{\text {loc }}([0, T] \times \mathbb{R}), \quad h\left(0, x_{2}\right)=0 .
$$


Similarly, the general solution $\pi \in C\left([0, T], L_{\mathrm{loc}}^{\infty}\left(\mathbb{R}^{N}\right) w *\right)$ to (1.6) (i.e. $\pi \in$ $\mathcal{W}^{T}$ ), is given by

$$
\begin{aligned}
\pi\left(t, x_{1}, x_{2}\right)= & \mathbb{1}_{\left|x_{1}\right| \geq T-t} \psi\left(\left(\left|x_{1}\right|-(T-t)\right)_{+} \operatorname{sgn} x_{1}, x_{2}\right) \\
& +\mathbb{1}_{\left|x_{1}\right|<T-t} g\left(\left(T-t-\left|x_{1}\right|\right)_{+}, x_{2}\right) \operatorname{sgn} x_{1},
\end{aligned}
$$

with

$$
\psi \in L_{\mathrm{loc}}^{\infty}(\mathbb{R} \times \mathbb{R}), \quad g \in L_{\mathrm{loc}}^{\infty}([0, T] \times \mathbb{R}) .
$$

Now, we see from (6.2) that $p \in \mathcal{E}^{T}$ if and only if $\varphi \equiv 0$, and therefore

$$
V_{e}^{T}=\left\{\left(t, x_{1}, x_{2}\right) \in(0, T) \times \mathbb{R}^{2} \text { such that }\left|x_{1}\right|<T-t\right\} .
$$

Then, we see that $\pi$ in (6.4) lies in $\mathcal{V}^{T}$ if and only if $g \equiv 0$.

Let us now look for transport flows $X^{T}$, as in Definition 3.5. Each component of $X^{T}$ has to be of the form (6.2), with final data $\varphi_{i}$ given respectively by $\varphi_{1}\left(x_{1}, x_{2}\right)=x_{1}$ and $\varphi_{2}\left(x_{1}, x_{2}\right)=x_{2}$. The functions $h_{i}$ are arbitrary, thus

$$
\begin{aligned}
X^{T}\left(t, x_{1}, x_{2}\right)= & \left(\left(\left|x_{1}\right|-(T-t)\right)_{+} \operatorname{sgn} x_{1}+h_{1}\left(\left(T-t-\left|x_{1}\right|\right)_{+}, x_{2}\right),\right. \\
& \left.x_{2}+h_{2}\left(\left(T-t-\left|x_{1}\right|\right)_{+}, x_{2}\right)\right),
\end{aligned}
$$

and we need only to write that $J\left(X^{T}\right) \geq 0$. According to the conditions (6.3), in the set where $\left|x_{1}\right| \geq T-t$ we have $J\left(X^{T}\right)=1$, while $J\left(X^{T}\right)=$ $-\left(\operatorname{sgn} x_{1}\right)\left(\partial_{t} h_{1}\left(1+\partial_{2} h_{2}\right)-\partial_{2} h_{1} \partial_{t} h_{2}\right)\left(\left(T-t-\left|x_{1}\right|\right)_{+}, x_{2}\right)$ in the set $\left|x_{1}\right|<T-$ $t$. Since this last quantity is odd with respect to $x_{1}$, its nonnegativity implies that it vanishes identically, thus the condition $J\left(X^{T}\right) \geq 0$ resumes to

$$
\partial_{t} h_{1}\left(1+\partial_{2} h_{2}\right)-\partial_{2} h_{1} \partial_{t} h_{2} \equiv 0 .
$$

Once this is satisfied, we have

$$
J\left(X^{T}\right)=\mathbb{1}_{\left|x_{1}\right| \geq T-t},
$$

and as predicted by Lemma 3.4, this is independent of the choice of $h_{1}, h_{2}$. According to Definition 3.9, $\pi$ in (6.4) is reversible if and only if $g \equiv 0$. Thus for our coefficient a, we have here equality in (3.18).

Finally, we can observe that the transport flow is not unique even if we impose the semi-group property. Denoting by $X(s, t, x)=X^{s}(t, x)$ for $0 \leq t \leq$ $s \leq T$, this means that

$$
X(s, t, X(t, \tau, x))=X(s, \tau, x) \text { for } \quad 0 \leq \tau \leq t \leq s \leq T .
$$

Choosing $h_{1} \equiv 0$ and $h_{2}\left(t, x_{2}\right)=\lambda t$ in (6.7), the conditions (6.3) and (6.8) are satisfied thus we get a transport flow $X_{\lambda}$ for any $\lambda \in \mathbb{R}$,

$$
X_{\lambda}\left(s, t, x_{1}, x_{2}\right)=\left(\left(\left|x_{1}\right|-(s-t)\right)_{+} \operatorname{sgn} x_{1}, x_{2}+\lambda\left(s-t-\left|x_{1}\right|\right)_{+}\right) .
$$

Noticing that $\left(\tau_{1}-\left|x_{1}\right|\right)_{+}+\left(\tau_{2}-\left(\left|x_{1}\right|-\tau_{1}\right)_{+}\right)_{+}=\left(\tau_{1}+\tau_{2}-\left|x_{1}\right|\right)_{+}$for any $\tau_{1}, \tau_{2} \geq 0$, one easily checks that (6.10) holds for any $\lambda$. However, it is wellknown that for a coefficient satisfying the OSLC condition, there is only one flow $X$ which solves $\partial_{s} X(s, t, x)=\mathbf{a}(s, X(s, t, x))$ in the sense of Filippov. It is indeed obtained for the choice $\lambda=0$. 


\section{Appendix}

This appendix is devoted to some useful results on Jacobian determinants. The first two lemmas are proved in [6], respectively Lemma 2.5 and Theorem 2.4.

Lemma A.1. (Divergence chain rule.) Let $g \in C^{1}\left(\mathbb{R}^{d}, \mathbb{R}^{d}\right)$ such that

$$
\operatorname{div} g \in L^{\infty}, \quad|g(y)| \leq C(1+|y|) .
$$

Let $\Omega$ be an open subset of $\mathbb{R}^{N}$, and $u \in L_{\mathrm{loc}}^{1}\left(\Omega, \mathbb{R}^{d}\right), d \leq N$, such that $\partial_{x_{1}} u, \cdots$, $\partial_{x_{N}} u \in L_{\text {loc }}^{d}\left(\Omega, \mathbb{R}^{d}\right)$. Then for any injective $\sigma: \mathbb{N}_{d} \rightarrow \mathbb{N}_{N}$,

$$
\begin{gathered}
\sum_{k=1}^{d} \frac{\partial}{\partial x_{\sigma(k)}}\left[I\left(\left[\frac{\partial u_{i}}{\partial x_{\sigma(j)}}\right]_{1 \leq i, j \leq d}\right) g(u)\right]_{k} \\
=(\operatorname{div} g) \circ u \operatorname{det}\left[\frac{\partial u_{i}}{\partial x_{\sigma(j)}}\right]_{1 \leq i, j \leq d},
\end{gathered}
$$

where $I(A)$ denotes the pseudo inverse (i.e. transpose of the cofactor matrix) of $A \in \mathbb{M}_{d}(\mathbb{R}), I(A)=(\operatorname{com} A)^{t}$.

Lemma A.2. Let $\Omega$ be an open subset of $\mathbb{R}^{N}$ and $u \in L_{\text {loc }}^{1}\left(\Omega, \mathbb{R}^{d}\right), d \leq N$, such that $\partial_{x_{1}} u, \cdots, \partial_{x_{N}} u \in L_{\text {loc }}^{d}\left(\Omega, \mathbb{R}^{d}\right)$. Then for any Borel set $Z \subset \mathbb{R}^{d}$ such that $|Z|=0$ and any one-to-one $\sigma: \mathbb{N}_{d} \rightarrow \mathbb{N}_{N}$,

$$
\mid\left\{x \in \Omega ; u(x) \in Z \text { and } \operatorname{det}\left(\frac{\partial u_{i}}{\partial x_{\sigma(j)}}\right)_{1 \leq i, j \leq d} \neq 0\right\} \mid=0 .
$$

In other words, $\operatorname{rank}(D u(x))<d$ almost everywhere on $u^{-1}(Z)$.

Lemma A.3. Let $\Omega$ be an open subset of $\mathbb{R}^{N+1}$ with $N \geq 1$, and $H_{1}, \ldots, H_{N} \in$ $L_{\mathrm{loc}}^{1}(\Omega)$ such that $\nabla H_{i} \in L_{\mathrm{loc}}^{N}(\Omega)$. Define for $k=0, \ldots, N$

$$
V_{k}=(-1)^{k} \operatorname{det}\left(\frac{\partial H_{i}}{\partial x_{j}}\right)_{i=1, \ldots, N, j=0, \ldots, k-1, k+1, \ldots, N}
$$

Then $V \in L_{\text {loc }}^{1}(\Omega)$ satisfies $\operatorname{div} V=0$ in the sense of distributions in $\Omega$.

Proof. Let us assume that $H_{i} \in C^{\infty}$, the general case being easily deduced by approximation. Consider an arbitrary function $H_{0} \in C^{\infty}$, and apply Lemma A.1 with $d=N+1, g(y)=(1,0, \ldots, 0) \in \mathbb{R}^{N+1}, u=\left(H_{0}, \ldots, H_{N}\right)$, and $\sigma=$ Id. We get that $\operatorname{div}(I(\nabla u) g)=0$, which gives the result since $V=(-1)^{N} I(\nabla u) g$. 
We can notice in the previous lemma that $H_{i}$ are like Hamiltonians for $V$ since $V \cdot \nabla H_{i}=0$. This can be seen by the identity

$$
\begin{aligned}
V \cdot \nabla H_{i} & =(-1)^{N}[I(\nabla u) g] \cdot \nabla H_{i} \\
& =(-1)^{N} \operatorname{det}\left(\nabla H_{1}, \ldots, \nabla H_{N}, \nabla H_{i}\right)=0 .
\end{aligned}
$$

Another proof of Lemma A.3 is to consider the $N$ differential form $\omega=$ $d H_{1} \wedge \cdots \wedge d H_{N}$. Then its external differential vanishes, $d \omega=0$, because $d^{2}=0$. One can check that $\omega=\sum_{k=0}^{N}(-1)^{k-1} V_{k} d x_{0} \wedge \cdots \wedge \widehat{d x_{k}} \wedge \cdots \wedge d x_{N}$, giving $\operatorname{div} V=0$.

The next lemma is a generalization of the weak stability of the Jacobian determinant (see [29, 30] and [5]).

Lemma A.4. Let $\Omega$ be an open subset of $\mathbb{R}^{N}$ and $1 \leq d \leq N$. Consider $a$ sequence $u^{(n)}$ bounded in $L_{\text {loc }}^{1}\left(\Omega, \mathbb{R}^{d}\right)$ such that $\partial_{x_{j}} u^{(n)}$ is bounded in $L_{\text {loc }}^{d}\left(\Omega, \mathbb{R}^{d}\right)$ for $1 \leq j \leq N$, and assume that $u^{(n)} \rightarrow u$ in $L_{\text {loc }}^{1}\left(\Omega, \mathbb{R}^{d}\right)$, with $\partial_{x_{j}} u \in L_{\text {loc }}^{d}\left(\Omega, \mathbb{R}^{d}\right)$. Consider also a sequence $\psi_{n}$ bounded in $L^{\infty}\left(\mathbb{R}^{d}\right)$ such that $\psi_{n} \rightarrow \psi$ in $L^{\infty} w *$. Then for any $\sigma: \mathbb{N}_{d} \rightarrow \mathbb{N}_{N}$ one-to-one,

$$
\psi_{n}\left(u^{(n)}\right) \operatorname{det}\left[\frac{\partial u_{i}^{(n)}}{\partial x_{\sigma(j)}}\right]_{1 \leq i, j \leq d} \rightarrow \quad \psi(u) \operatorname{det}\left[\frac{\partial u_{i}}{\partial x_{\sigma(j)}}\right]_{1 \leq i, j \leq d}
$$

in the sense of distributions in $\Omega$.

Proof. According to Lemma A.2, both sides in (A.6) are well-defined as elements of $L_{\text {loc }}^{1}(\Omega)$.

Let us consider first the case $\psi_{n}=\psi=1$, i.e. the case of pure Jacobians. Then the result can be established by induction on $d$. When $d=1$, this is obvious by linearity. Assuming the result to be true at level $d-1$, with $2 \leq d \leq N$, we apply Lemma A.1 with $g(y)=y / d$, to $u^{(n)}$ and $u$. We deduce that we only need to prove that

$$
I\left(A_{n}\right) u^{(n)} \rightarrow I(A) u,
$$

where

$$
A_{n}=\left[\frac{\partial u_{i}^{(n)}}{\partial x_{\sigma(j)}}\right]_{1 \leq i, j \leq d}, \quad A=\left[\frac{\partial u_{i}}{\partial x_{\sigma(j)}}\right]_{1 \leq i, j \leq d} .
$$

Since the coefficients of $I\left(A_{n}\right)$ are sub-determinants of order $d-1$ of $A_{n}$, the induction assumption ensures that $I\left(A_{n}\right) \rightarrow I(A)$. Since $I\left(A_{n}\right)$ is bounded in $L_{\text {loc }}^{d^{\prime}}$, this convergence holds in $L_{\text {loc }}^{d^{\prime}}-w *$. But according to Sobolev imbedding, $u^{(n)} \rightarrow u$ in $L_{\text {loc }}^{d}$, and this yields (A.7).

In the case $\psi \neq \equiv 1$, define

$g(y)=\frac{1}{\left|S^{d-1}\right|} \int_{\mathbb{R}^{d}}\left(\frac{y-z}{|y-z|^{d}}+\frac{z}{|z|^{d}}-\mathbb{1}_{|z| \geq 1}\left(\frac{y}{|z|^{d}}-d \times(y \cdot z) \frac{z}{|z|^{d+2}}\right)\right) \psi(z) d z$.

Then $g \in C_{\mathrm{loc}}^{0, \beta}\left(\mathbb{R}^{d}, \mathbb{R}^{d}\right)$ for any $\beta<1$, div $g=\psi$, and

$$
|g(y)| \leq C_{d}\left(1+|y| \ln ^{+}|y|\right)\|\psi\|_{\infty} .
$$


This last estimate can be obtained as follows. For $R>0$, define $M_{R}$ to be the supremum over $|y| \leq R$ of the $L^{1}$ norm in $z$ of the term between parentheses in (A.9). Then one can check easily that $M_{2 R} \leq 2 M_{R}+C_{d} R$, and this implies that $M_{R} \leq C_{d}\left(1+R \ln ^{+} R\right)$. This proves (A.10). Now, we claim that (A.2) holds with this nonlinearity $g$. Indeed the right-hand side makes sense according to Lemma A.2, and the left-hand side also by (A.10), since by Sobolev imbedding, $u \in L_{\text {loc }}^{d+\varepsilon}$ for some $\varepsilon>0$. The validity of (A.2) for $g$ defined by (A.9) and $\psi \in L^{\infty}\left(\mathbb{R}^{d}\right)$ can be obtained by approximating $\psi$ by some smooth functions with compact support.

Finally, for our stability result when $\psi_{n} \rightarrow \psi$ in $L^{\infty} w *$, according to the previous argument, we can apply (A.2) to $u$ and $g$ corresponding to $\psi$, and also to $u^{(n)}$ and $g_{n}$ corresponding to $\psi_{n}$. Thus in order to get (A.6), it is enough to prove that $I\left(A_{n}\right) g_{n}\left(u^{(n)}\right) \rightarrow I(A) g(u)$, with $A_{n}$ and $A$ defined by (A.8). By the first step on pure Jacobians, we have $I\left(A_{n}\right) \rightarrow I(A)$ in $L_{\text {loc }}^{d^{\prime}}-w *$. But since $\psi_{n} \rightarrow \psi$ we have $g_{n} \rightarrow g$ locally uniformly. Using (A.10) and the fact that $u^{(n)} \rightarrow u$ in $L_{\text {loc }}^{d+\varepsilon}$ for some $\varepsilon>0$, we get that $g_{n}\left(u^{(n)}\right) \rightarrow g(u)$ in $L_{\mathrm{loc}}^{d}$, and thus $I\left(A_{n}\right) g_{n}\left(u^{(n)}\right) \rightarrow I(A) g(u)$.

\section{References}

[1] G. AlBERTI, Rank-one properties for derivatives of functions with bounded variation, Proc. Roy. Soc. Edinburgh, Sect. A 123 (1993), 239-274.

[2] G. Alberti and L. Ambrosio, A geometric approach to monotone functions in $\mathbb{R}^{n}$, Math. Z. 230 (1999), 259-316.

[3] L. Ambrosio, Transport equation and Cauchy problem for BV vector fields, Invent. Math. 158 (2004), 227-260.

[4] J.-P. Aubin and A. Cellina, "Differential Inclusions", Springer-Verlag, 1984.

[5] J. BALL, Convexity conditions and existence theorems in nonlinear elasticity, Arch. Ration. Mech. Anal. 42 (1977), 337-403.

[6] F. Bouchut, Renormalized solutions to the Vlasov equation with coefficients of bounded variation, Arch. Ration. Mech. Anal. 157 (2001), 75-90.

[7] F. Bouchut and L. Desvillettes, On two-dimensional Hamiltonian transport equations with continuous coefficients, Differential Integral Equations 14 (2001), 1015-1024.

[8] F. BouchUT and F. JAMES, One-dimensional transport equations with discontinuous coefficients, Nonlinear Anal. 32 (1998), 891-933.

[9] F. Bouchut and F. JAmes, Differentiability with respect to initial data for a scalar conservation law, In: "Hyperbolic problems: theory, numerics, applications", Vol. I (Zürich, 1998), 113-118, Internat. Ser. Numer. Math., Vol. 129, Birkhäuser, Basel, 1999.

[10] F. Bouchut and F. JAMES, Duality solutions for pressureless gases, monotone scalar conservation laws, and uniqueness, Comm. Partial Differential Equations 24 (1999), 2173 2189.

[11] E. D. Conway, Generalized solutions of linear differential equations with discontinuous coefficients and the uniqueness question for multidimensional quasilinear conservation laws, J. Math. Anal. Appl. 18 (1967), 238-251. 
[12] C. M. DAfERmos, Generalized characteristics and the structure of solutions of hyperbolic conservation laws, Indiana Univ. Math. J. 26 (1977), 1097-1119.

[13] R. J. DiPeRnA and P.-L. Lions, Ordinary differential equations, transport theory and Sobolev spaces, Invent. Math. 98 (1989), 511-547.

[14] A. F. FILIPPOV, Differential equations with discontinuous right-hand side, Amer. Math. Soc. Transl. (2) 42 (1964), 199-231.

[15] A. F. FILIPPOv, Differential equations with discontinuous righthand sides, In: "Coll. Mathematics and Its Applications", Kluwer Academic Publishers Dordrecht-Boston-London, 1988.

[16] E. Godlewski, M. Olazabal and P.-A. Raviart, On the linearization of hyperbolic systems of conservation laws. Application to stability, In: "Équations aux dérivées partielles et applications", articles dédiés à J.-L. Lions, Gauthier-Villars, Paris, 1998, 549-570.

[17] E. Godlewski, M. Olazabal and P.-A. Raviart, On the linearization of systems of conservation laws for fluids at a material contact discontinuity, J. Math. Pures Appl. (9) 78 (1999), 1013-1042.

[18] M. Hauray, On two-dimensional Hamiltonian transport equations with $L_{\mathrm{loc}}^{p}$ coefficients, Ann. Inst. H. Poincaré Anal. Non Lin. 20 (2003), 625-644.

[19] D. HofF, The sharp form of Oleinik's entropy condition in several space variables, Trans. Amer. Math. Soc. 276 (1983), 707-714.

[20] F. JAMES and M. SEPÚlvedA, Convergence results for the flux identification in a scalar conservation law, SIAM J. Control Optim. 37 (1999), 869-891.

[21] B. L. Keyfitz and H. C. KRANTZER, A strictly hyperbolic system of conservation laws admitting singular shocks, In :"Nonlinear Evolution Equations that Change Type", IMA Volumes in Mathematics and its Applications, Vol. 27, 1990, Springer-Verlag, New-York, 107-125.

[22] P. LeFLOCH, An existence and uniqueness result for two nonstrictly hyperbolic systems, In: "Nonlinear Evolution Equations that Change Type", IMA Volumes in Mathematics and its Applications, Vol. 27, 1990, Springer-Verlag, New-York, 126-138.

[23] C. T. McMullen, Lipschitz maps and nets in Euclidean space, Geom. Funct. Anal. 8 (1998), 304-314.

[24] O. A. Oleinik, Discontinuous solutions of nonlinear differential equations, Amer. Math. Soc. Transl. (2), 26 (1963), 95-172.

[25] B. Popov and G. Petrova, Linear transport equations with discontinuous coefficients, Comm. Partial Differential Equations 24 (1999), 1849-1873.

[26] B. Popov and G. Petrova, Linear transport equations with $\mu$-monotone coefficients, J. Math. Anal. Appl. 260 (2001), 307-324.

[27] F. Poupaud and M. RASCLE, Measure solutions to the linear multidimensional transport equation with discontinuous coefficients, Comm. Partial Differential Equations 22 (1997), 337-358.

[28] T. QIN, Symmetrizing nonlinear elastodynamics system, J. Elasticity 50 (1998), 245-252.

[29] Y. ReshetNyAK, On the stability of conformal mappings in multidimensional spaces, Sibirsk. Mat. Zh. 8 (1967), 91-114.

[30] Y. RESHETNYAK, Stability theorems for mappings with bounded excursions, Sibirsk. Mat. Zh. 9 (1968), 667-684. 
[31] D. H. Wagner, Conservation laws, coordinate transformations, and differential forms, In: "Hyperbolic Problems: Theory, Numerics, Applications", J. Glimm, J. W. Grove, M. J. Graham, B. J. Plohr (Eds.), World Scientific, Singapore, New Jersey, London, Hong Kong, 1996, 471-477.

[32] Y. ZHENG and A. MAJDA, Existence of global weak solutions to one-component VlasovPoisson and Fokker-Planck-Poisson systems in one space dimension with measures as initial data, Comm. Pure Appl. Math. 47 (1994), 1365-1401.

DMA, Ecole Normale Supérieure et CNRS 45 rue d'Ulm

75230 Paris cedex 05, France

Francois.Bouchut@ens.fr

Laboratoire MAPMO, UMR 6628

Université d'Orléans

45067 Orléans cedex 2, France

Francois.James@univ-orleans.fr

Laboratoire J.-L. Lions, UMR 7598

Université Pierre et Marie Curie, BP 187

4 place Jussieu

75252 Paris cedex 05, France

current address:

Laboratoire MAPMO, UMR 6628

Université d'Orléans

45067 Orléans cedex 2, France

Simona.Mancini@univ-orleans.fr 